
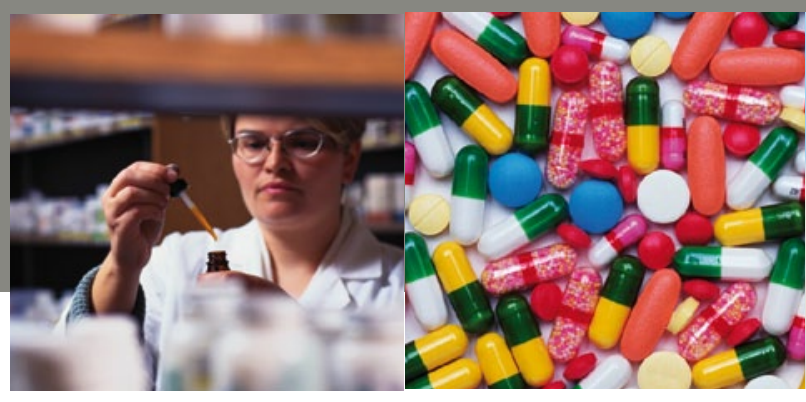

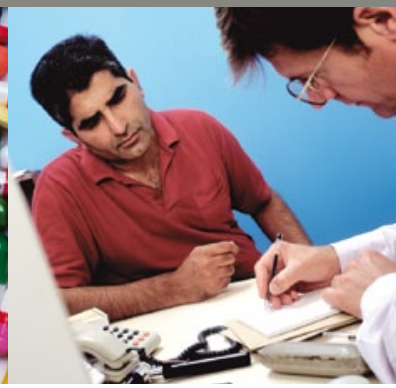

Was ist neu in der medikamentösen Therapie?

Wir halten Sie auf dem Laufenden mit Berichten von Kongressen und Symposien der pharmazeutischen Industrie.

Keine Anämie, keine erkennbare klinische Ursache

\section{Eisensubstitution bessert auch in diesem Fall die Fati- guesymptomatik}

- Eine Studie konnte zeigen, dass eine Eisensubstitution bei nicht anämischen Frauen mit unerklärter Fatigue positive Effekte hat. Eisenmangel kann sich in einer Anämie ( $\mathrm{Hb}<12 \mathrm{~g} / \mathrm{dl}$ ) äußern. In den letzten Jahren ist aber auch der sog. prälatente Eisenmangel ohne bisher aufgetretene Anämie in das Blickfeld der Forschung gerückt. Dabei kann es bereits bei Ferritinwerten unter $50 \mu \mathrm{g} / \mathrm{l}$ und normalen $\mathrm{Hb}$-Werten zu therapiebedürftigen Beschwerden (z. B. Müdigkeit, Antriebslosigkeit, verminderte Konzentrationsfähigkeit und reduzierte Muskelleistung) kommen.

\section{Anstieg der Hämoglobin- und Ferritinwerte}

In einer multizentrischen, doppelblinden, placebokontrollierten randomisierten Studie gingen Vaucher et al. (CMAJ 2012. DOI: 10.1503/cmaj.110950) der Frage nach, wie die orale Eisensubstitution die Fatiguesymptomatik, Lebensqualität und Blutparameter von 198 prämenopausalen Frauen (1853 Jahre alt) mit Eisenmangel ohne Anämie und unerklärter Fatigue beeinflusst. Alle Frauen hatten "normale" Hämoglobinwerte von mindestens $12 \mathrm{~g} / \mathrm{dl}$, niedrige Ferritinspiegel von unter $50 \mu \mathrm{g} / \mathrm{l}$ und litten unter unerklärter Fatigue (d.h. für die Symptome bestanden keine offensichtlichen klinischen Gründe wie z. B. Schwangerschaft oder Stillzeit, psychiatrische, rheumatische, onkologische oder kardio- vaskuläre Erkrankungen etc.).

Die Frauen erhielten zwölf Wochen lang einmal täglich $80 \mathrm{mg}$ Eisen(II)-sulfat (Tardyferon ${ }^{\circledR}$ ) oder Placebo. Primärer Endpunkt der Studie war eine Besserung der Fatiguebeschwerden gemessen anhand eines Patienten-Fragebogens (Current and Past Psychological Scale (CAPPS)). Nach der zwölfwöchiger Behandlung zeigte sich, dass sich die Fatiguesymptomatik unter der Eisensubstitution signifikant verbessert hatte: Der Fatigue-Score (CAPPS) nahm in der Verumgruppe $(n=96)$ um $47,7 \% a b$, in der
Placebogruppe um 28,8\%. Auch weitere Parameter für Fatigue (MAF-Scale) verbesserten sich unter der Eisentherapie. Bezüglich der Lebensqualität, Depression und Ängstlichkeit ergaben sich keine Unterschiede. Darüber hinaus zeigte sich bereits nach sechs Wochen eine deutliche Verbesserung der Blutwerte in der Verumgruppe. Hämoglobin- und Ferritinwerte stiegen signifikant an, auch der Hämatokrit sowie die Transferrin-Sättigung nahmen zu.

- Red.

Quelle: Nach Informationen von Pierre Fabre

\title{
Aktiv für ältere Patienten
}

\section{Förderpreis SilverStar wird erneut verliehen}

In Würde altern, wünscht sich jeder Mensch. Gesundheit und ein selbstbestimmtes Leben gehören dazu. Dass ein erfülltes Leben auch für ältere Menschen mit Diabetes möglich bleibt, ist das Ziel des SilverStar - ein Preis, mit dem Berlin-Chemie Projekte oder Initiativen fördert, die zu einer besseren Versorgung älterer Menschen mit Diabetes beitragen. Getreu dem Motto „Aktiv für ältere Patienten“ kann sich jeder darum bewerben - ob Gesundheitsprofis (wie z. B. Ärzte, Pflegekräfte, Apotheker) oder engagierte Angehörige, Freunde, Nachbarn oder selbst Betroffene: Gesucht werden kreative Ideen, die älteren Menschen mit Diabetes den Umgang mit ihrer Erkrankung erleichtern.

Der Name steht symbolisch für die Generation der "Silberhaarigen", d.h. für ältere Menschen mit Diabetes. Sie sind die Stars, sie sollen im Mittelpunkt stehen.

Diabetes kann für viele ältere Menschen zum Problem werden, denn er kann die Lebensqualität beeinträchti- gen. Der SilverStar will zeigen, dass es sich lohnt, den Kopf nicht in den Sand zu stecken. Wer aktiv bleibt und positiv denkt, kann viel erreichen. Das gilt nicht nur für die Betroffenen selbst, sondern auch für die Menschen im sozialen Umfeld, die sich aktiv für ältere Menschen mit Diabetes einsetzen.

Die geriatrische Diabetestherapie wird in den nächsten Jahren weiter an Bedeutung gewinnen und zu einer der größten Herausforderungen werden. Bereits jetzt unterstützt das Unternehmen zahlreiche Projekte in den Bereichen Diabetes und Altersmedizin. Mit dem Preis, der 2013 zum dritten Mal verliehen wird, setzt das Unternehmen ein weiteres Zeichen und fördert mit einer Gesamtsumme von 25000 Euro Projekte, die zu einer Verbesserung der Versorgung und zu einer Erhöhung der Lebensqualität älterer Menschen mit Diabetes beitragen. Infos und Bewerbung unter: www.silverstar-preis.de

\footnotetext{
- Red.

Quelle: www.silverstar-preis.de
} 